\title{
Correspondence:
}

\section{Difference between how ambulance service personnel use paper and electronic patient care records when attending older people at home}

Marina BUSWELL, Jane FLEMING, Philip LUMBARD, Larissa S PROTHERO, Sarah AMADOR and Claire GOODMAN

\section{Corresponding Author:}

Dr Marina Buswell, Research Associate, NIHR CLAHRC-EoE, Institute of Public Health, Forvie Site, University of Cambridge School of Clinical Medicine, Box 113 Cambridge Biomedical Campus, Cambridge, CB2 0SR, UK, mb934@medschl.cam.ac.uk, +44 (0) 7780 452214

\section{Source of funding}

The research was funded by the National Institute for Health Research (NIHR) Collaboration for Leadership in Applied Health Research and Care East of England at Cambridgeshire and Peterborough NHS Foundation Trust. The views expressed are those of the authors and not necessarily those of the NHS, the NIHR or the Department of Health.

\section{Conflicts of interest}

None declared

Key Words

Medical records

Electronic health records

Data Collection

Emergency ambulance

Older people

Emergency transport

Ambulance services 


\section{Difference between how ambulance service personnel use paper and electronic patient care records when attending older people at home}

In the course of our study, Research into Older people with Dementia and their carers' use of Emergency ambulance Services (RODES), we have noted a difference between how ambulance service personnel use paper-based as opposed to electronic patient care records (PCRs) when attending older people. Looking at 373 PCRs for patients aged 65 years and over (187 electronic, 186 paper) we found that fewer than one in twenty patients were reported as 'treated and discharged' (otherwise known as 'left at scene') in the electronic PCR group compared with more than one third in the paper PCR group. Conversely, the proportion of patients in the electronic PCR group reported as treated and transported was markedly higher (almost 85\%) compared to only half in the paper PCR group. This clearly has important implications for anyone seeking to use ambulance service PCRs to measure older people's hospital 'transport' rates by emergency ambulance crews, both within Trusts where more than one PCR format is used and for comparison of findings from areas with different record systems.

This analysis was conducted on the basis of anecdotal evidence from a RODES stakeholder meeting where it was noted that if ambulance crews were 'leaving someone at home' then they would be more likely to use a paper PCR rather than the electronic PCR, in part to leave the patient and carers with a copy of the care record.

To test this observation we revisited an audit of non-patient-identifiable data for 373 patients aged 65 and over that had been completed in the last 12 months and found this confirmed the observation. Of the 187 electronic PCRs, 158 were treated and transported and just nine were treated and discharged, three were deceased and not transported and seven required no treatment. Of the 186 paper PCRs 96 were treated and transported and 67 were treated and discharged, eleven were deceased and not transported and one required no treatment. The remaining 21 records (10 electronic and 11 paper) had various 'other' outcomes (Table $1)$. 
Table 1 Outcomes reported in a sample of electronic and paper-based PCRs for patients aged 65 and over

\begin{tabular}{|l|c|c|}
\hline Electronic PCR & $\mathbf{n = 1 8 7}$ & $\%(95 \% \mathrm{Cl})$ \\
\hline Deceased \& Not Transported & 3 & $1.6 \%(-0.2-3.4)$ \\
No Treatment Required & 7 & $3.7 \%(1.0-6.5)$ \\
\hline Treated \& Discharged & $\mathbf{9}$ & $\mathbf{4 . 8 \% ( 1 . 7 - 7 . 9 )}$ \\
\hline Treated \& Transported & 158 & $\mathbf{8 4 . 5 \% ( 7 9 . 3 - 8 9 . 7 )}$ \\
\hline Other & 10 & $5.3 \%(2.1-8.6)$ \\
\hline Paper PCR & $\mathbf{n = 1 8 6}$ & $\%(95 \% \mathrm{Cl})$ \\
\hline Deceased \& Not Transported & 11 & $5.9 \%(2.5-9.3)$ \\
No Treatment Required & 1 & $0.5 \%(-0.5-1.6)$ \\
\hline Treated \& Discharged & $\mathbf{6 7}$ & $\mathbf{3 6 . 0 \% ( 2 9 . 1 - 4 2 . 9 )}$ \\
\hline Treated \& Transported & 96 & $\mathbf{5 1 . 6 \% ~ ( 4 4 . 4 - 5 8 . 8 ) ~}$ \\
\hline Other & 11 & $5.9 \%(2.5-9.3)$ \\
\hline
\end{tabular}

We acknowledge that we have only tested this observation in one opportunistic and small sample. However, we felt that the difference was so striking that it is worth bringing to the attention of researchers and commissioners who may be using these data sources to inform decisions.

This finding may also have implications for the roll-out of electronic PCRs in ambulance services if there is not the facility to leave a copy of the PCR with the patient and their carers. We hope this observation is useful in alerting PCR data users of the need for care, and awareness of the context in which these data sources are derived, and thank our colleague for pointing out how different record-keeping methods may be used in practice. 